\title{
Quit for keeps: tailored smoking cessation guides for pregnancy and beyond
}

\author{
Victor J Strecher, Kathy R Bishop, Jay Bernhardt, John M Thorp, Brian Cheuvront, \\ Paul Potts
}

Although health care providers appear to be an obvious choice for delivering smoking cessation education, they often lack the resources, training, and time to provide anything more than a recommendation to quit and generic pamphlets for reinforcement. Traditionally, this type of material is designed to include information for many potential users, thus making it difficult for an individual to find the pieces most relevant to them. In contrast, tailored print materials provide only information which is relevant to a subject, making it far more usable. One study assessing tailored print messages found a threefold increase in cessation rates among patients in a family practice setting, four months after receipt of tailored versus untailored smoking cessation messages. ${ }^{1}$ Numerous other studies, targeting a number of different populations, have also shown that tailored messages are an effective intervention for smoking cessation. ${ }^{2}$

In tailoring, we use individual patient responses to select only relevant behaviour change messages. These messages provide information specific to an individual's needs and interests and can reinforce messages from health professionals. Because they are personalised, tailored materials offer a potentially superior alternative to generic materials designed to reach a broad audience. Quit for Keeps used a pre-test/post-test experimental design to test the effects of tailored interventions on pregnant smokers.

\section{Methods}

Participants in this study included 92 women enrolled at the Taubman obstetrics and gynaecology (ob/gyn) clinic at the University of Michigan and 81 women enrolled at the ob/gyn clinic at the University of North Carolina hospital from December 1996 to December 1997. Eligible participants were those who reported having smoked at least 100 cigarettes in their lifetime and who were either still smoking or had quit since becoming pregnant. Subjects were screened for eligibility during the New to the Nurse orientation program in North Carolina or during their first pre-natal visit to the clinic in Michigan. Potential subjects were given a hand held computer (Apple Newton MessagePad) and asked to fill out a short screening survey. After the computer determined that a subject was eligible, she was then approached by a research assistant and asked to participate in the project. Consenting women were then given the hand held computer for a second time and asked to complete the baseline questionnaire which assessed smoking status and asked questions on behavioural and psychosocial variables, including: stages of change for quitting smoking, perceived benefits of and barriers to quitting, self efficacy, and demographic information. These constructs were used to create the tailored messages for those subjects randomised to the experimental condition. A urine sample was collected at baseline to confirm smoking status, and values $<80 \mathrm{ng} / \mathrm{ml}$ were considered indicative of abstinence.

Subjects were then randomised via computer algorithm and assigned to either the control or experimental condition. Subjects in the experimental condition $(n=88)$ received a series of tailored smoking cessation messages through the mail, one after each pre-natal visit during which a follow up questionnaire was completed. Subjects in the control condition ( $\mathrm{n}=85$ ) received $A$ pregnant woman's guide to quit smoking after the first visit and no other materials.

At each subsequent prenatal visit, women were again given the hand held computer and asked to complete a short follow up interview. The follow up interviews were used to assess a subject's progress toward quitting and any changes in barriers or benefits that could be addressed by the tailored messages. Urine samples were collected at the prenatal visit, during the 24th week of pregnancy, and again at the six week postpartum visit. Final data were collected, by telephone, at three months postpartum for evaluation purposes.

Data were transferred from the hand held computers to a desktop computer. Data collected from subjects in the experimental condition were used to tailor a pregnancy guide and quit plan, which was mailed to their home. The guides included smoking cessation information, tips and strategies, and pictures and information about the developing fetus, tailored to the length of gestation. Each time a subject in the experimental group filled out a new interview during a prenatal visit, a new guide was sent with updated information.

\section{Results}

Baseline comparisons of women in the tailored and untailored materials conditions revealed no significant differences in age, race, education, number of cigarettes smoked before pregnancy, and baseline stage of change (table 1). In order to examine the primary hypothesis of the study, we focused on postpartum, self reported cessation using an intent-to-treat model (treating women who were lost to follow up as smokers). In this model, 8 of the 87 $(9.2 \%)$ women who received untailored 
Table 1 Demographic, pregnancy, and smoking history information at first prenatal visit*

\begin{tabular}{lll}
\hline & $\begin{array}{l}\text { Experimental } \\
(n=88)\end{array}$ & $\begin{array}{l}\text { Control } \\
(n=85)\end{array}$ \\
\hline $\begin{array}{ll}\text { Demographics } \\
\text { Age (years) (mean (SD)) }\end{array}$ & $25.8(5.5)$ & $26.6(5.7)$ \\
Education (years) (mean (SD)) & $12.5(2)$ & $12.5(2.1)$ \\
Race (white) (n (\%)) & $76 / 87(87.4)$ & $69 / 85(81.2)$ \\
$\quad$ Ethnicity (non-Hispanic) (n (\%)) & $81 / 86(92)$ & $78 / 85(91.8)$ \\
Pregnancy history & $18.1(8.4)$ & $16(7.5)$ \\
Weeks pregnant (gestational week) (mean (SD)) & $30 / 63(47.6)$ & $23 / 61(37.7)$ \\
Primigravida (n (\%)) & $0.7(1.0)$ & $0.9(0.8)$ \\
Prior births (mean (SD)) & & \\
Smoking information & $15.1(3.5)$ & $15.7(3.4)$ \\
Age started (years) (mean (SD)) & $20.3(11)$ & $18.7(9.6)$ \\
Cigs smoked/day prepregnancy (mean (SD)) & $12.9(12.2)$ & $11.8(14)$ \\
Cigs smoked/day during past week (mean (SD)) & $2701.8(3459.3)$ & $2597(2899.5)$ \\
Cotinine concentration (ng/ml) (mean (SD)) & $1.7(0.5)$ & $1.6(0.5)$ \\
Number of prior quit attempts (mean (SD)) & $1.0(1.2)$ & $1.1(1.9)$ \\
Number of other smokers in household (mean (SD)) & $55 / 66(83.3)$ & $45 / 59(76.3)$ \\
Planning to quit within next 30 days (n (\%)) & $58 / 73(79.5)$ & $55 / 69(79.7)$ \\
Thinking about quitting during pregnancy (n (\%)) & &
\end{tabular}

^ No significant differences were found between conditions at baseline.

messages (control condition) quit smoking, while 10 of the $104(9.6 \%)$ women who received tailored messages (experimental condition) quit smoking. This difference was not significant. Excluding subjects who were lost to follow up increased rates of cessation to $14 \%(8 / 57)$ in the control condition and $15.2 \%$ $(10 / 104)$ in the experimental condition; this difference remained non-significant. There were also no differences between control and experimental group cessation rates at mid term. Additionally, no differences in the number of cigarettes smoked per day existed between the tailored and untailored groups, when comparing baseline and mid term, baseline and postpartum, or mid term and postpartum data. These same comparisons were made for changes in cotinine concentration and, again, no significant difference was found. Finally, after adjusting for multiple comparisons, significant differences in quit rates, smoking reduction rates, or cotinine concentrations did not exist between control and experimental conditions, even when stratified by age, race, education, number of cigarettes smoked at baseline, stage of change, or study site (University of North Carolina versus University of Michigan).

After completion of the research project, staff conducted telephone interviews at both sites with a total of 19 experimental and nine control group women to assess the participant's appraisal of aspects of the project. Overall, $90 \%$ of the subjects felt that the hand held computer was "very easy" to use and 90\% felt "very comfortable" using a computer to answer difficult questions. Of the women in the intervention group, most (55\%) considered the information they received to be useful, all felt that the materials addressed their reasons for smoking, $60 \%$ felt that their needs were addressed, and almost $70 \%$ believed their barriers were addressed in the information.

\section{Discussion}

In this pilot study, the tailored message intervention produced no apparent change in subjects' smoking behaviour over and above an untailored self help guide. The study had a low power to detect differences because of the fact that approximately $10 \%$ of the women screened were found eligible for the study, in contrast to the expected $30 \%$, which resulted in a smaller number of women in the trial. The difficulties in subject recruitment are most likely attributed to two factors. First, the initial estimate of the percentage of women eligible for the study was based simply on the smoking prevalence rate in women in the state of Michigan, rather than on actual smoking prevalence among patients at the two ob/gyn facilities (as this information was not available). Second, it is unknown how many eligible women answered the screening questions in such a way as to make themselves appear ineligible. Since eligibility was dependent entirely on self report smoking status, many eligible woman may have opted out of the study by incorrectly answering the screening questions. However, the similarity between outcomes in the experimental and control groups suggests that a larger sample size would not have detected a benefit in favour of the tailored intervention.

We are therefore perplexed by the findings which are counter to the majority of published or reported tailored message trials. ${ }^{3-5}$ It is possible that a different style or sequence of the tailored materials might have had a different impact or that different theoretical models should be used in developing messages. Women with very low readiness to change were recruited along with women who were prepared to quit $(67.6 \%)$, which may have also affected the efficacy of the intervention. It is also possible that women during pregnancy are less receptive to materials sent by mail than they would be to materials delivered in some other manner (such as by the physician). During the evaluation of the participant's satisfaction with the project, no clear suggestions for improvement emerged. Instead, a diverse group of comments were collected and ranged from being very happy with the materials and having successfully quit, to being uninterested in the project and having no interest in the materials at all. What is clear is that further studies are necessary in order to evaluate more fully the use of tailored smoking cessation materials for pregnant women.

Special thanks are extended to all of the women who participated in this project, both at the University of Michigan and at the University of North Carolina, and the clinic staff at both locations. We would also like to acknowledge the hard work of the following individuals for carrying out the day to day research activities: John Brusk, MPH; Eduardo Alvarado, MPH; Dana Santana, MPH; and Chittra Chaivorapol, MPH. Finally, a thank you to Andrew to Doug Wolf, PhD for data analysis.

1 Strecher VJ, Kreuter M, Den Boer DJ, et al. The effects of computer-tailored smoking cessation messages in family practice settings [see comments]. I Fam Pract 1994; 39:262-70.

2 Strecher VJ. Computer-tailored smoking cessation materials: a review and discussion. Patient Education and Counseling. 1999;36:107-17.

3 Orleans CT, Boyd NR, Noll E, Crosette L. Intervening through a prescription benefit plan for nicotine patch users. Paper presented at the Society of Behavioral Medicine Annual Meeting. Washington DC, March 1996.

4 Prochaska JO, DiClemente CC, Velicer WF, Rossi JS Standardized, individualized, interactive and personalized self-help programs for smoking cessation. Health Psychol 1993;12:399-405

5 Shiffman S, Gitchell J, Strecher V. Real-world efficacy of computer-tailored smoking cessation material as a supplement to nicotine replacement. 10th Conference on Tobacco or Health, Beijing, August 1997. 